\title{
Etude de la production d'hydrogène en bioréacteur par une bactérie photosynthétique Rhodobacter capsulatus 1. Photobioréacteur et conditions optimales de production d'hydrogène Hydrogen production in bioreactor by a photosynthetic bacterium Rhodobacter capsulatus 1 . Photobioreactor and optima conditions of hydrogen production
}

\author{
S. Delachapelle, M. Renaud et P. M. Vignais
}

Volume 4, numéro 1, 1991

URI : https://id.erudit.org/iderudit/705091ar

DOI : https://doi.org/10.7202/705091ar

\section{Aller au sommaire du numéro}

\section{Éditeur(s)}

Université du Québec - INRS-Eau, Terre et Environnement (INRS-ETE)

ISSN

0992-7158 (imprimé)

1718-8598 (numérique)

\section{Découvrir la revue}

\section{Citer cet article}

Delachapelle, S., Renaud, M. \& Vignais, P. M. (1991). Etude de la production d'hydrogène en bioréacteur par une bactérie photosynthétique Rhodobacter capsulatus 1. Photobioréacteur et conditions optimales de production d'hydrogène. Revue des sciences de l'eau / Journal of Water Science, 4(1), 83-99. https://doi.org/10.7202/705091ar
Résumé de l'article

Un réacteur de 10 litres, automatisé pour la culture continue en anaérobiose de bactéries photosynthétiques, a été réalisé et mis au point. Ce réacteur parfaitement agité a été utilisé dans différentes conditions de fonctionnement en système fermé (batch), en système semi-ouvert (atouts de substrats concentrés en discontinu), en système ouvert (chémostat) avec et sans recyclage de la biomasse, afin d'étudier la consommation, par les bactéries, d'un substrat carboné, le lactate. La production d'hydrogène par la bactérie photosynthétique Rhodobacter capsulatus, souche B10, résultant de la dégradation du lactate, est optimale pour des cultures en continu diluées, limitées en source azotée. Ainsi, à un taux de dilution de $0,04 \mathrm{~h}^{-1}$, avec $5 \mathrm{mM}$ glutamate dans le milieu nutritif, la densité bactérienne étant de 2,1 è $660 \mathrm{~nm}$, on a observé une production continue moyenne de $65 \mathrm{ml} \cdot \mathrm{h}^{-1} \cdot \mathrm{l}^{-1}$ pendant une période de 200 heures. Pour des concentrations bactériennes élevées, la limitation d'énergie lumineuse entrain une perte d'activité nitrogénase et, de ce fait, une chute de la production d'hydrogène. 


\title{
Etude de la production d'hydrogène en bioréacteur par une bactérie photosynthétique Rhodobacter capsulatus
}

\section{Photobioréacteur et conditions optimales} de production d'hydrogène

\author{
Hydrogen production in bioreactor by a photosynthetic \\ bacterium Rhodobacter capsulatus
}

1. Photobioreactor and optima conditions of hydrogen production

S. DELACHAPELLE1, M. RENAUD22 et P. M. VIGNAIS 3

Reçu le 30 janvier 1989, accepte pour publication le 19 septembre $1990^{*}$

RÉSUMÉ

Un réacteur de 10 litres, automatisé pour la culture continue en anaéroblose de bactéries photosynthétiques, a été réalisé et mis au point. Ce réacteur parfaitement agité a été utilisé dans différentes conditions de fonctionnement en système fermé (batch), en système semi-ouvert (ajouts de substrats concentrés en discontinu), en système ouvert (chémostat) avec et sans recyclage de la biomasse, afin d'étudier la consommation, par les bactéries, d'un substrat carboné, le lactate. La production d'hydrogène par la bactérie photosynthétique Rhodobacter capsulatus, souche B10, résultant de la dégra dation du lactate, est optimale pour des cultures en continu diluées, limittées en source azotée. Ainsi, à un taux de dilution de $0,04 \mathrm{~h}^{-1}$, avec $5 \mathrm{mM}$ glutamate dans le millieu nutritif, la densité bactérienne étant de 2,1 a $660 \mathrm{~mm}$, on a observé une production continue moyenne de $85 \mathrm{ml}^{-\mathrm{h}^{-1}} \cdot \mathrm{t}^{-1}$ pendant une période de $\mathbf{2 0 0}$ heures. Pour des concentrations bactériennes élevées, la limitation d'énergie lumineuse entraine une perte d'activité nitrogénase et, de ce fait, une chute de la production d'hydrogène.

Mots cles : Rhodobacter capsulatus, degradation du lactate, photobiorbacteur, production d'hydrogene.

Abréviations: ATP : adenosine triphosphate ; ADP : adénosine diphosphate : Pi : orthophosphate.

SUMMARY

A photobioreactor was set up to cultivate a photosynthetic bacterium in continuous cultures. The bioreactor was designed so as to 1) allow the capture of light energy by bacteria through a spiral transparent flexlble tube placed under the light, in a water bath maintaining the growth temperature at

1. Adresse actuelle : Sociête $3 M$ France, nue Geneviève Couturier, BP 324, 92506 Rueil Malmaison.

2. Laboratoire de Génie des Procédés Papetier (CNRS URA 1100), EFPG, BP 65, 38402 Saint-Martin-d'Héres.

3. Laboratoire de Biochimis Microbienne (CNRS URA 1130 alliée à l'INSERM), CENG, BP $85 \mathrm{X}, 38041$ Grenoble Cedex.

* Les commentaires seront reçus jusqu'au 30 octobre 1991. 
$30^{\circ} \mathrm{C}$; 2) male the suspension of bacterla circulate continuously in the reactor with volumetric pump to maintain the medium hompeneous; 3) allow degassing of the suspension in a degassing chamber ; 4) feed the culture with nutritive media, add neutralizing solution ( $\mathrm{pH} 7$ ) and withdrax aliquots while malntaining constant the volume of the culture ; 5) recycle the bacteria by filtration when the bioreactor was used as a closed system (batch).

The photosyntbotic bacterlum was Rhodobacter capsulatus strain B10 is known to be a good $\mathrm{H}_{2}$ producer [Hillmer and Gest (1977) J. Bacteriol. 129, 724-731]. The bioreactor was run using $10 \mathrm{I}$ of a synthetic medium contalining factate as carbon soures and glutamate as nitrogen sounce. It was studied for Its capacity to degrade lactato. Glutamate was the growth-liliting substrate allowing a maximum derepresslon of nitrogenase, the enzyme catalysing the reduction of protons to $\mathrm{H}_{2}$. The bacterial suspension was continuousiy circulated in the photoreactor, concelved as a plane light captor of $1 \mathrm{~m}^{2}$, to avoid bacterial self-shading and affow regeneration of ATP by photophosphorylation at high rates. The circult was tightiy closed to avoid air entry, which would prevent $\mathrm{H}_{2}$ production due to respiration of the bacteria.

To run it under automated conditions, the bioreactor was equipned with two temperature sensors, two pH electrodes, a water level detector, a manometer and a computer-controlled electric valve. The bioreactor, of the well-mixed type, was used under various working conditions, namely as a closed (batch) system, as a fed-batch system (discontinuous additions of concentrated substrates), and as an open system (chemostat) with or without biomass recycling.

Control of key parameters (pH, temperature, dilution rates) allowed us to define the culture conditions producing maximal amounts of molecular hydrogen. The production of $\mathrm{H}_{2}$ accompanying lactate degradation was maxlmal in diluted nltrogen-limited continuous cultures. It was observed at a dilution rate of $0.04 \mathrm{r}^{-1}$, with $5 \mathrm{~mm}$ glutamate in the influent medium, the optical density of the culture being 2.1 at $660 \mathrm{am}$. Under these conditions an average production $\mathrm{H2}$ of $85 \mathrm{ml} \cdot \mathrm{h}^{-1} \cdot \mathrm{I}^{-1}$ was observed over a $200 \mathrm{~h}$ period. At higher bacterial concentrations, the limitation of light energy resuited in a decrease in nitrogenase activity and therefore in a drop in the production of hydrogen.

The interdependence of various parameters $(\mathrm{pH}$, dilution rates, $\mathrm{N}$ and $\mathrm{C}$ sources light intensity) renders the system complex and not easily controlted by computer. Indeed, we observed that during recycling of the bacteria by the use of an ultrafiltration cell, the bacteria became a fermentative-type of metabolism accompanied by a decrease in nitrogenase activity and therefore in a drop in the production of bydrogen.

Key-words : Rhodobacter capsulatus, lactate degradation, photobioreactor, hydrogen production.

\section{INTRODUCTION}

Les bactéries photosynthétiques ont la capacité de réduire les protons de l'eau en hydrogène moléculaire. Le complexe enzymatique qui catalyse cette réaction est la nitrogénase (HILLMER et GEST, 1977 ; MEYER et al., 1978b ; WEAVER et al., 1980) qui, en présence d'azote atmosphérique et en absence d'oxygène, peut aussi réduire $\mathrm{N}_{2}$ en ammoniac selon la réaction :

$$
\mathrm{N}_{2}+8 \mathrm{e}^{-}+8 \mathrm{H}^{+}+\mathrm{nATP} \longrightarrow 2 \mathrm{NH}_{3}+\mathrm{H}_{2}+\mathrm{nADP}+\mathrm{nPi}
$$


La réaction catalysée par la nitrogénase nécessite de l'ATP et un donneur d'électrons à bas potentiel. Les bactéries photosynthétiques peuvent utiliser l'énergie lumineuse pour resynthétiser rapidement l'ATP, par photophosphorylation de l'ADP, de sorte que la production d'hydrogène par ces bactéries paraît dépendante de la lumière (voir par exemple VINCENZINI et al., 1982). En absence d'azote, tous les électrons sont utilisés par la nitrogénase pour réduire les protons et former de l'hydrogène (revues par MEYER et al., 1978b; VIGNAIS et al., 1981, 1985 ; WILLISON et al., 1983). La bactérie photosynthétique Rhodobacter capsulatus (autrefois appelée Rhodopseudomonas capsulata, IMHOFF et al., 1984) peut synthétiser de grandes quantités de l'enzyme nitrogénase à l'intérieur de la cellule - jusqu'à $25 \%$ du poids sec (JOUANNEAU et al., 1985) - et de ce fait, montre une forte capacité de production d'hydrogène (HILLMER et GEST, 1977 ; VIGNAIS et al., 1984 ; revue par HALLENBECK, 1983).

L'objectif de ce travail était de définir la configuration d'un photoréacteur permettant de maintenir $R$. capsulatus en culture, dans des conditions appropriées pour permettre, d'une part, l'élimination de substrats carbonés tels qu'on peut les trouver dans des effluents d'industries agroalimentaires et, d'autre part, une récupération d'énergie sous forme d'hydrogène. Dans cette étude, un photobioréacteur (assimilé à un réacteur idéal parfaitement agité) a été utilisé en système fermé et en système ouvert pour étudier la dégradation d'un milieu synthétique contenant du lactate et du glutamate, respectivement comme source de carbone et comme source d'azote nécessaires à la croissance des micro-organismes. Dans cet article, nous décrivons le réacteur qui a été utilisé pour cultiver $R$. capsulatus d'abord en batch, puis en chémostat, et les activités de production d'hydrogène lors de la dégradation du lactate observées dans des conditions de croissance bactérienne définies. Des réacteurs, de conception et de géométrie différentes, ont déjà été décrits dans la littérature pour cultiver ce type de bactéries (VINCENZINI et al., 1981 ; STEVENS et al., 1983 ; VGNAIS et al., 1984 ; JOUANNEAU ot al., 1986).

\section{MÉTHODES}

\section{Conditions de culture et mesure de la densité bactérienne}

Le milieu nutritif ( $\mathrm{pH} 3,0$ ou 6,9) est constitué du milieu minéral minimum décrit par WEAVER et al. (1975), supplémenté par du glutamate $(2,5$ ou $7 \mathrm{mM}$ ) comme source d'azote et du DL-lactate (25 ou $30 \mathrm{mM}$ ) comme source de carbone. II est stérilisé dans un récipient sous pression, une bonbonne Millipore, qui servira ensuite de nourrice d'alimentation sous pression d'argon. Le pH est maintenu à 7 en culture continue. La souche utilisée est la souche B10 de $\boldsymbol{R}$. capsulatus généreusement fournie par le Professeur H. GEST, Bloomington, USA. Dans les expériences de longue durée, afin de diminuer les risques de contamination par des bactéries sulfato-réductrices, le sulfate du milieu nutritif a été diminué et le magnésium apporté sous forme de 
chlorure de magnésium. Pour inhiber la croissance des bactéries sulfatoréductrices, des algues et des méthanogènes, on a quelquefois rajouté au milieu nutritif $2 \mathrm{mM}$ de molybdate et $10 \mathrm{mg} \cdot 1^{-1}$ de chloroforme ainsi que des extraits de houblons (humulone, lupulone et leurs isomères) a raison de 30 unités d'amertume, et parfois, I'herbicide chloroxuron jusqu'à une concentration de $10 \mathrm{ppm}$ qui renforce l'efficacité du houblon (SEGERS et VERSTRAETE, 1985a).

La densité bactérienne est évaluée par la mesure de l'absorbance à $660 \mathrm{~nm}$, réalisée à l'aide d'un spectrophotomètre LERES S67 (à $660 \mathrm{~nm}$ l'absorption due aux pigments est minimale). La concentration des bactéries est évaluée également par le poids sec bactérien (psb), obtenu après centrifugation (centrifugeuse SORVALL $20000 \mathrm{G}, 15 \mathrm{mn}$ ) de $10 \mathrm{ml}$ de culture bactérienne. Les cellules sont lavées avec de l'eau distillée, centrifugées de nouveau puis desséchées au four à $80^{\circ} \mathrm{C}$. Le poids $s e c$ bactérien ( $p s b)$ est exprimé en $\mathrm{g}^{\cdot \mathrm{I}^{-1}}$. Le psb et l'absorbance à $660 \mathrm{~nm}$ $\left(A_{660 \mathrm{~nm}}\right)$ chez $R$. capsulatus, sont liés par la relation : psb $\left(g \cdot \mathfrak{l}^{-1}\right)=$ $0,45 \mathrm{~A}_{660 \mathrm{~nm}}$ (JOUANNEAU, 1982). Le poids sec bactérien exprimé en gramme de carbone par litre est égal à la moitié de l'extrait sec (LEVEAU et BOUIX, 1982) soit psb de carbone $\left(g \cdot F^{-1}\right)=p s b\left(g \cdot F^{-1}\right) / 2$.

\section{Mesure de lactivité de la nitrogénase par réduction de l'acétylène en éthylène}

Les conditions, décrites par MEYER et al. (1978a), sont les suivantes. Des flacons de $12 \mathrm{ml}$, bouchés par des bouchons en caoutchouc à jupe rabattable, contenant $2 \mathrm{ml}$ de suspension bactérienne sous argon, sont agités dans un bain marie illuminé (5000 Lux) par le fond et thermostaté à $30^{\circ} \mathrm{C}$. Après $5 \mathrm{mn}$ d'équilibration, du lactate (concentration finale $20 \mathrm{mM}$ ) est ajouté puis, après $1 \mathrm{mn}$, un $\mathrm{ml}$ d'acétylène pur est injecté. Des fractions aliquotes de la phase gazeuse $(50 \mu \mathrm{l})$ sont prélevées en fonction du temps et l'éthylène formé est dosé par chromatographie gazeuse dans un chromatographe Intersmat IGC 121 DFL équipé d'un détecteur à ionisation de flamme; la colonne d'acier de $1,5 \mathrm{~m}$ de long sur $3,2 \mathrm{~mm}$ de diamètre est remplie de Porapak R (80-100 mesh); le gaz vecteur est l'azote $\left(60 \mathrm{ml} \cdot \mathrm{mn}^{-1}\right)$ et la température du four $70^{\circ} \mathrm{C}$. Une unité d'activité nitrogénase vaut 1 nmole $\mathrm{C}_{2} \mathrm{H}_{4} \cdot \mathrm{h}^{-1} \cdot \mathrm{mg}^{-1} \mathrm{psb}$.

\section{Dosage de l'hydrogène par chromatographie gazeuse}

L'incubation se fait dans les mêmes conditions que pour la mesure d'éthylène mais sans addition d'acétylène dans la phase gazeuse. $\mathrm{H}_{2}$ produit est mesuré dans des fractions aliquotes de la phase gazeuse de $50 \mu l$ injectées dans un chromatographe Intersmat $120 \mathrm{MB}$, équipé d'une colonne Porapak $Q$ (80-100 mesh, $2 \mathrm{~m} \times 3,2 \mathrm{~mm})$ et d'un catharomètre, et calibré par des injections de quantités connues de $\mathrm{H}_{2}$ (COLBEAU et al., 1980). Le gaz vecteur est largon. 


\section{Dosage du lactate et des acides gras à courte chaîne}

Ces mesures sont réalisées par chromatographie liquide haute performance (CLHP) à l'aide d'une chaîne WATERS en système isocratique (pompe $6000 \mathrm{~A}$, débit $0,6 \mathrm{~m} \cdot \mathrm{mn}^{-1}$; éluant : acide sulfurique $0,01 \mathrm{~N}$ ). Les différents acides sont séparés sur une colonne du type Aminex lon Exclusion HPX 87 H (BIO-RAD Labs). La longueur d'onde de détection est à $210 \mathrm{~nm}$. Les suspensions bactériennes sont centrifugées (centrifugeuse Eppendorf) pendant $5 \mathrm{mn}$, le surnageant est récupéré, filtré sur Millipore $0,22 \mu \mathrm{m}$ et passé en CLHP. Le lactate et les acides détectés sont séparés et dosés quantitativement par intégration des pics.

\section{MATÉRIEL}

\section{Réalisation du photobioréacteur}

Les fonctions principales du photobioréacteur sont : 1) de permettre le captage, par les bactéries, de l'énergie lumineuse grâce à une tubulure transparente exposée à la lumière et refroidie pour maintenir la température de croissance à $30^{\circ} \mathrm{C}$; 2) de faire circuler la suspension bactérienne, à l'aide d'une pompe volumétrique adaptée, pour assurer l'homogénéisation du milieu ; 3) de permettre le dégazage de la suspension dans un bac de récupération du gaz produit ; 4) d'alimenter les bactéries en milieu nutritif (influent) et en solutions de neutralisation $(\mathrm{pH}=7)$, et de soutirer la suspension de manière à garder un volume constant; et 5) de filtrer la biomasse dans le cas d'un fonctionnement du réacteur avec recyclage de bactéries.

Le schéma de principe est illustré sur la figure 1. Le photoréacteur est constitué d'une tubulure transparente en PVC de $30 \mathrm{~m}$ de long (diamètre intérieur $15 \mathrm{~mm}$, volume 5,3l) enroulée en spirale sur une surface plane où l'éclairement est maximum. C'est dans cette tubulure que circulent les bactéries. La tubulure en spirale formant un capteur plan d'environ $1 \mathrm{~m}^{2}$ est illuminée par deux lampes placées a environ $80 \mathrm{~cm}$ au-dessus du plan, soit des lampes à iode 500 ou $1000 \mathrm{~W}$, intensité 20000 ou 40000 lumens, soit des lampes à vapeur de sodium haute pression, puissance $150 \mathrm{~W}$, intensité de 15000 lumens. La spirale est complètement immergée dans un bac de refroidissement en inox avec circulation d'eau refroidie dans un cryostat, le débit d'eau est réglable manuellement par une vanne à pointeau. II est à noter que lorsque des lampes à vapeur de sodium sont utilisées, le refroidissement de la spirale n'est pas nécessaire. Les bactéries circulantes passent dans un bac cylindrique en plexiglas appelé bac de dégazage pour la récupération du gaz (volume 4,6 I). Ce bac est rempli aux $2 / 3$ de suspension bactérienne ; il est fermé par un couvercle serré par un joint torique muni d'embouts et de vannes manuelles et d'un septum pour injection de produits et prise d'échantillons gazeux. Ce bac est parfaitement étanche. 


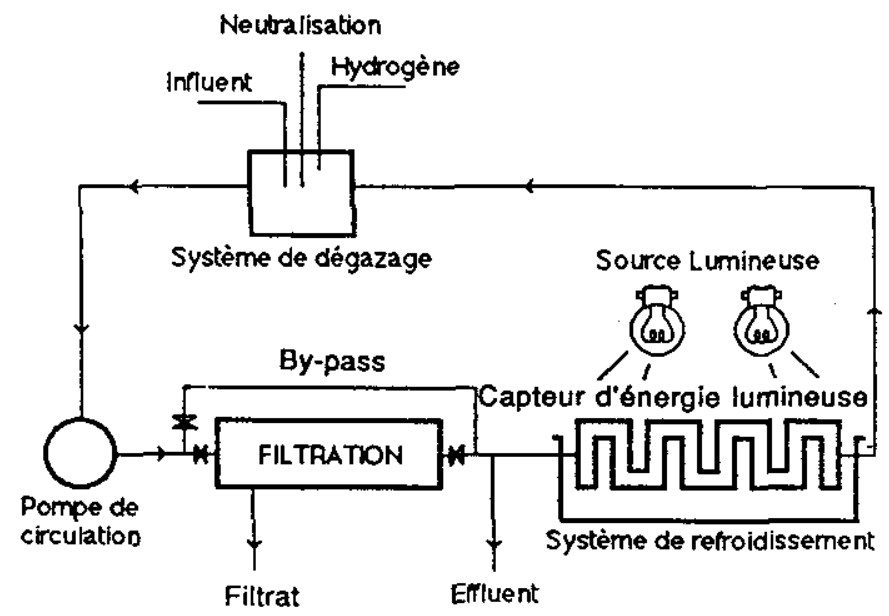

Figure 1 Schéma du réacteur.

Le circuit comprend deux cellules de fitration tangentielle permettant la séparation bactéries/milieu épuré, un * by-pass ^ est aménagé pour un fonctionnement du réacteur sans filtration. Chaque unité de filtration est constituée par une unité inox Carbosep avec 6 tubes utilisables, de surface filtrante importante, qui peut supporter des températures supérieures à $100^{\circ} \mathrm{C}$ et est donc facilement stérilisable. (A noter qu'une cellule de microfiltration tangentielle utilisant une membrane nucléopore en polycarbonate a été testée et s'est révélée être inutilisable du fait de son colmatage rapide).

La circulation de la suspension bactérienne dans le photoréacteur est assurée par une pompe à membrane Téflon, parfaitement étanche, qui n'entraîne aucun effet de cisaillement sur les bactéries. La chambre de circulation du milieu est séparée de la partie mécanique du moteur, la pompe fonctionne par pulsations amorties par l'intermédiaire d'un amortisseur à soufflet et a fonctionné en continu pendant un an sans avoir nécessité le remplacement de la membrane. Le photoréacteur est alimenté en milieu nutritif, stérile et maintenu sous atmosphère d'argon, à l'aide de pompes à piston munies d'un cadenceur permettant de faire varier les débits d'alimentation de 0 a $10 \mathrm{l} \cdot \mathrm{h}^{-1}$. Ces pompes, commandées électriquement, se sont révélées être résistantes et fiables dans le temps.

Afin de diminuer la contamination par des micro-organismes de l'environnement, lors du stockage du milieu nutritif, ce dernier a été préparé à pH 3 , puis neutralisé par de la soude $\mathbf{N}$. L'apport de milieu nutritif et de soude s'est fait dans le bac de dégazage muni d'une sonde de niveau et d'une sonde de $\mathrm{pH}$. Les échantilions de culture pour analyses sont prélevés dans le bac de dégazage.

Le gazomètre est formé d'un récipient fermé dont le fond est percé d'un orifice et le couvercle formé d'une membrane souple en caoutchouc fixée sur le pourtour du récipient. Une tige métallique traversant de part et d'autre le récipient est fixée par une extrémité à la membrane, l'autre extrémité libre 
obstrue l'orifice quand la membrane est à l'état de repos. Le gazomètre est au départ rempli d'eau. Quand il y a production gazeuse, une légère surpression soulève la membrane libérant le pointeau de son orifice pour laisser s'écouler une certaine quantité d'eau jusqu'au rétablissement de la pression d'équilibre, qui est la pression atmosphérique. Le volume d'eau ainsi écoulé correspond au volume de gaz produit.

\section{Régulation et automatisme}

Le circuit est doté de capteurs. Deux capteurs de température ont été utilisés : un capteur à semi-conducteur (référence LM 335 de * National Semi-Conductor w) placé au centre de la tubulure, en contact avec la suspension bactérienne et une sonde de température en platine placée dans le bac de dégazage loin de la source de lumière; cette sonde a donné des mesures fiables.

Pour mesurer et réguler le $\mathrm{pH}$, une électrode de $\mathrm{pH}$ combinée a été placée au niveau des introductions de solutions dans le bac de dégazage ; mais la mesure dérive au cours du temps, par encrassement de l'électrode dû à des dépôts de matière biologique. Deux électrodes pressurisées avec de l'air comprimé (une de référence, l'autre de mesure) ont été placées dans une chambre de mesure en fin de circuit, avant le bac de dégazage. Ces électrodes ont donné entière satisfaction en donnant la mesure exacte du pH de la suspension avec un temps de réponse correspondant au temps de circulation du milieu et une précision de $\pm 0,01 \mathrm{pH}$. Le système de pressurisation évite l'encrassement des électrodes.

La mesure de niveau a été faite de deux façons : une mesure de niveau par barrière infrarouge (IR) (émetteur IR, TIL 31 et récepteur IR, TIL 81 de Texas Instrument) et une mesure à l'aide d'une sonde capacitive de niveau VEGA reliée à un transmetteur indicatif capacitif de niveau; cette sonde placée dans le bac de dégazage assure une régulation analogique indépendante de la pression du milieu.

La mesure des débits gazeux a été faite soit à l'aide d'un manomètre, placé en sortie du bac de dégazage, et d'une électrovanne commandée par ordinateur, soit à l'aide du gazomètre décrit plus haut qui donne une mesure précise adaptée pour de faibles débits. Les données des capteurs ont été stockées dans un micro-ordinateur GOUPIL 3.

Tout en suivant le même schéma de principe au cours de notre étude, nous avons réalisé deux versions de l'installation, l'une orientée sur une conduite automatique centralisée par ordinateur en introduisant une série d'électrovannes, l'autre orientée sur des systèmes d'asservissements autonomes en remplaçant les électrovannes par des pompes commandées et des vannes.

Plusieurs raisons sont à l'origine de la modification de la première version du réacteur en une deuxième version beaucoup plus simplifiée au niveau des automatismes avec laquelle nous avons poursuivi nos expériences :

- le système d'exploitation (MS DOS) du calculateur utilisé dans la première version était monotâche et son langage (BASIC) ininterruptible, ce qui entraînait une discontinuité dans la surveillance du procédé. 
- L'interaction des éléments capteurs, liaisons, cartes électroniques et électrovannes rendait le pilotage du procédé complexe. Si l'un des éléments présentait une défaillance, tout le système était bloqué, y compris le programme.

En conséquence, nous avons préféré utiliser des systèmes plus fiables, mieux maîtrisés, c'est-à-dire un régulateur analogique propre à chaque paramètre à réguler et indépendant des autres. Dans ce montage, l'ordinateur est conservé pour l'acquisition des données et pourra être utilisé dans l'avenir pour piloter les points de consigne (système en supervision). Nous avons pu ainsi réaliser des cultures en continu pendant deux mois en maîtrisant bien le procédé.

\section{RÉSULTATS}

\section{Fonctionnement en réacteur fermé}

Le réacteur est alimenté avec 10 litres de milieu nutritif (contenant lactate $25 \mathrm{mM}$, glutamate $5 \mathrm{mM}$ ) ensemencés avec une préculture de $R$. capsulatus. Les bactéries sont cultivées à la lumière de lampes à vapeur de sodium haute pression, en anaérobiose et à $30^{\circ} \mathrm{C}$. La croissance bactérienne comporte les phases caractéristiques d'une croissance en milieu non renouvelé (fig. 2). L'excrétion d'acides organiques (formiate-acétate) est liée à la dégradation du lactate mais celui-ci est toujours en excès dans le milieu. La croissance cellulaire est limitée par la concentration en glutamate ; la concentration cellulaire, en tin de croissance, augmente pour une teneur en substrat azoté plus élevée (fig. 3).

Dans un procédé utilisant un réacteur à biomasse en suspension, la capacité de traitement de linstallation est fonction de la concentration de la population bactérienne. Afin d'obtenir une densité bactérienne maximale, nous avons ajouté, à intervalles réguliers, un concentré de substrat de manière à rétablir les concentrations initiales des différents constituants du milieu, soit $25 \mathrm{mM}$ de lactate, $5 \mathrm{mM}$ de glutamate et les sels minéraux du milieu minimal (fig. 4). L'ajout de glutamate (substrat limitant) fait redémarrer la croissance et la densité de la suspension peut atteindre une valeur élevée d'absorbance $\left(A_{660} \mathrm{~nm}=12\right)$ qui correspond à une densité bactérienne de $5,4 \mathrm{~g}$ de bactéries par litre de milieu. Cette valeur n'est pas limitative, la culture ayant été volontairement interrompue, ce qui laisse prévoir que la multiplication cellulaire n'est pas inhibée par une modification du milieu.

Cependant, on constate lors des fortes concentrations bactériennes une baisse de lactivité de la nitrogénase, enzyme responsable de la formation d'hydrogène, qui provoque une baisse de la production $\mathrm{d}^{\prime} \mathrm{H}_{2}$. Cette baisse d'activité a pour origine la diminution de l'énergie lumineuse reçue par les bactéries. En effet, ces bactéries colorées en brun rendent les suspensions opaques quand elles sont nombreuses. Ainsi, les bactéries manquent de lumière et donc d'ATP nécessaire à la nitrogénase pour réduire les protons en 
$H_{2}$. A l'inverse, en suspension bactérienne diluée $\left(A_{660 \mathrm{~nm}}=2-3,5\right)$, on observe une forte activité de la nitrogénase et une bonne production gazeuse $\left(450 \mathrm{ml} \mathrm{H}_{2} \cdot \mathrm{h}^{-1}\right)$.

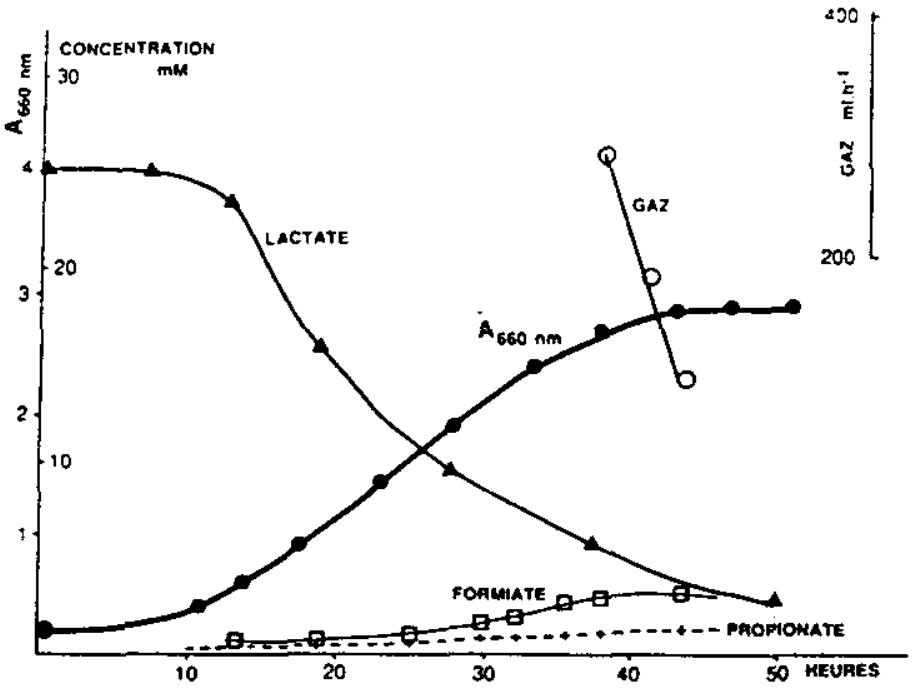

Figure 2 Culture batch de Rhodobacter capsulatus souche B10. Le milieu nutritif de départ contient lactate $25 \mathrm{mM}+$ glutamate $5 \mathrm{mM}$. Source lumineuse : 2 lampes a vapeur de sodium haute pression de $150 \mathrm{~W}$ chacune (15 000 lumens).

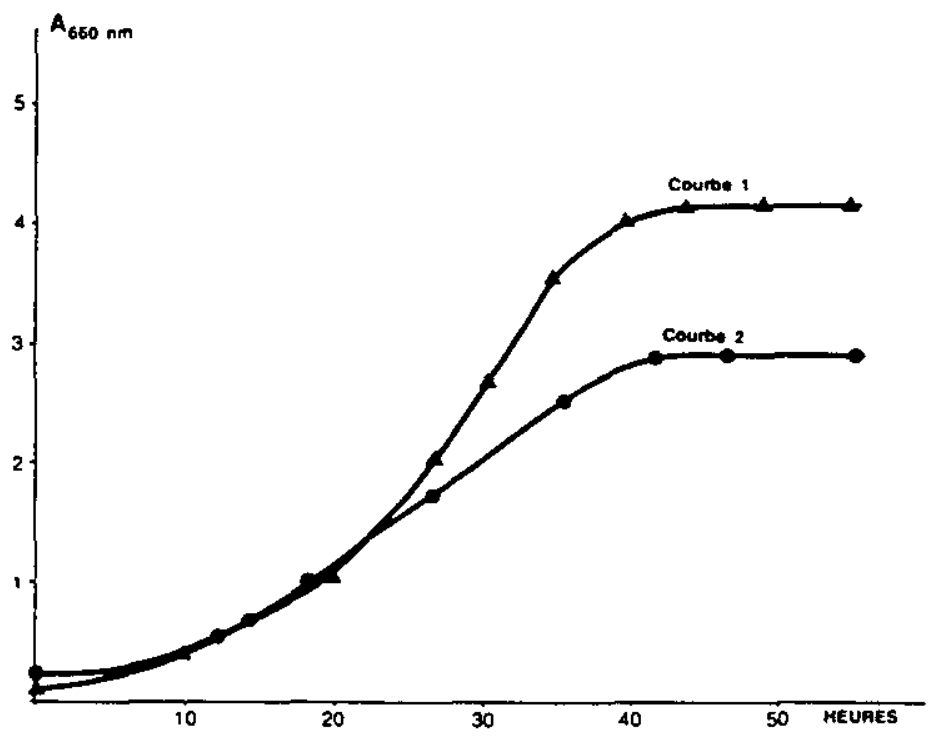

Figure 3 Variation de la densité bactérienne (A660 nm) en système batch dans deux milieux de culture différents: courbe 1: lactate $30 \mathrm{mM}+$ glutarnate $7 \mathrm{mM}$; courbe 2 : lactate $25 \mathrm{mM}+$ glutamate $5 \mathrm{mM}$. 


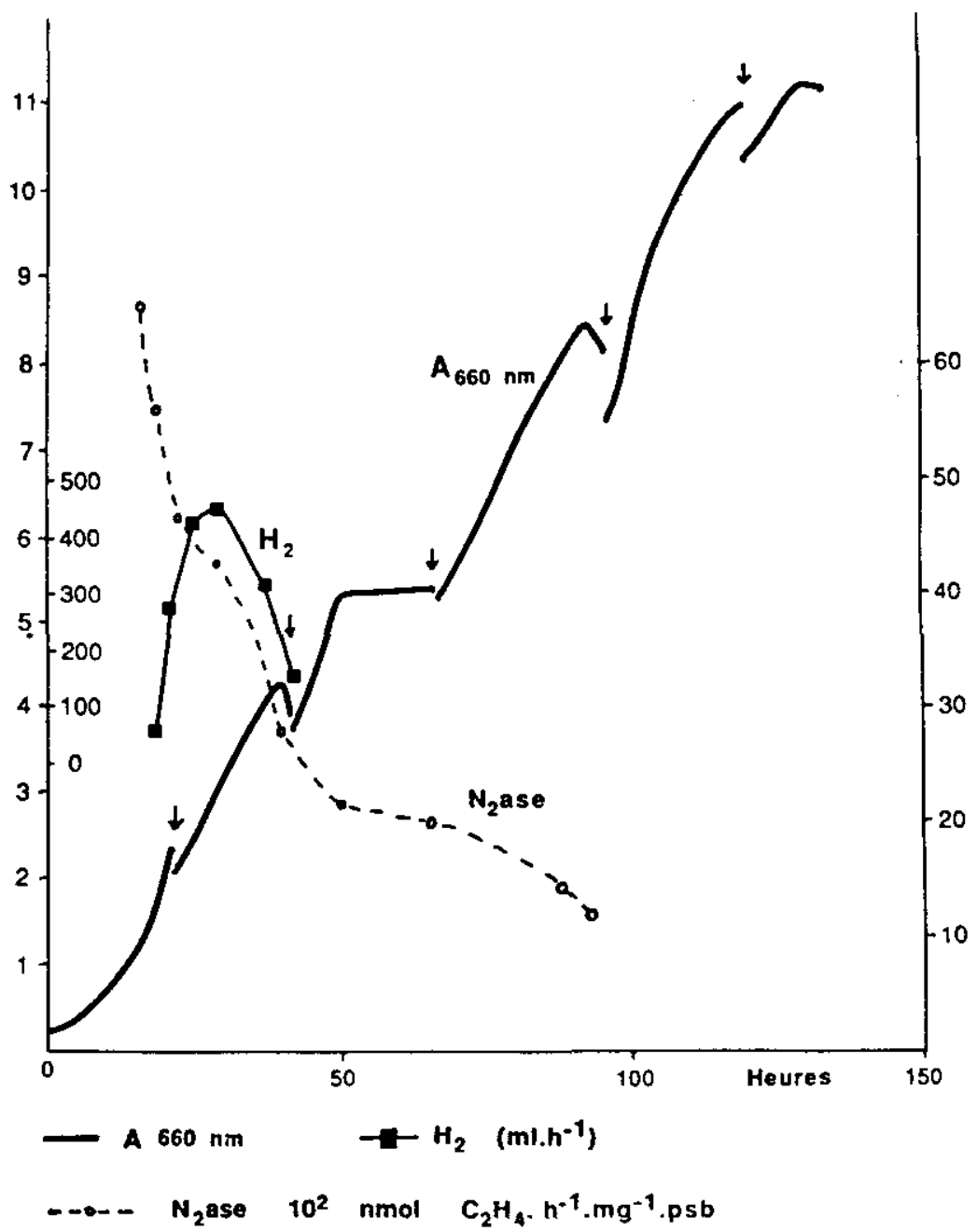

Figure 4 Culture en réacteur fermé avec ajouts successifs de milieu nutritif concentré $(\downarrow)$. Les concentrations initiales de lactate et de glutamate sont respectivement de $25 \mathrm{mM}$ et $5 \mathrm{mM}$. Evolution de la concentration bactérienne $\left(A_{660 \mathrm{~nm}}\right)$ de la production d'hydrogène $(--)$ et de l'activité nitrogénase (N2ase) (-O-) (voir Méthodes).

\section{Fonctionnement en réacteur ouvert}

La production d'hydrogène est favorisée par une faible population bactérienne et par une forte illumination. II faut cependant déterminer le taux de dilution (inverse du temps de passage global dans le réacteur) permettant d'optimiser la production d'hydrogène et la dégradation du lactate dans un système de culture en continu. 
Le tableau 1 met en évidence une diminution lente de la densité optique à l'équilibre au fur et à mesure que le taux de dilution augmente ( $D=0,02 \mathrm{~h}^{-1}$ a $\mathbf{D}=0,14 \mathrm{~h}^{-1}$ ). Cette diminution s'accompagne d'une augmentation de la concentration résiduelle de lactate dans la suspension et donc d'une réduction du taux de transformation du substrat carboné. La production d'hydrogène maximale pour un taux de dilution compris entre $0,03 \mathrm{~h}^{-1}$ et $0,04 \mathrm{~h}^{-1}$, chute et s'annule à $\mathrm{D}=0,12 \mathrm{~h}^{-1}$.

Ces résultats ont pu être confirmés par la mesure de l'absorbance à $660 \mathrm{~nm}\left(\mathrm{~A}_{660 \mathrm{~nm}}\right)$ dans des cultures en chémostat à différentes concentrations de lactate et de glutamate. La figure 5 permet d'établir une relation empirique entre l'absorbance $A_{660 \mathrm{~nm}}$, la concentration en glutamate $S_{0}$ (en $\mathrm{mM}$ ) introduite dans la solution, et le taux de dilution appliqué $D\left(e h^{-1}\right)$. La concentration résiduelle en glutamate étant dans tous les cas trop faible pour être mesurée, nous en concluons que cette espèce chimique est, pour les conditions étudiées, le substrat limitant. Cette relation empirique s'exprime de la façon suivante : $A_{660 \mathrm{~nm}}=0,9\left(S_{0}-1\right)\left[D_{\max } \cdot S_{0}-D\right]$ où $D_{\max }\left(e n h^{-1}\right)$ est le taux de dilution pour lequel la densité optique s'annule et qui est une fonction de la concentration en glutamate.

Le rapport de masse de matière vivante à la masse de lactate dégradé, c'est-à-dire le rendement sur lactate, est à peu près constant quel que soit le taux de dilution et le lactate est toujours en excès dans la suspension. La production d'hydrogène dépend, elle, du taux de dilution. Nous avons choisi le taux de dilution correspondant à une production gazeuse maximale, pour étudier la cinétique de la culture sur une période de 200 heures. L'état d'équilibre est atteint après $\mathbf{5 0}$ heures de culture. Au cours de la phase d'équilibre, le mécanisme de multiplication et de synthèses cellulaires fonctionne à une vitesse constante déterminée par le taux de ditution de la culture. Ainsi, pour une concentration en glutamate de $5 \mathrm{mM}$, la production d'hydrogène est en moyenne de $85 \mathrm{mi} \cdot \mathrm{h}^{-1} \cdot 1^{-1}$ correspondant à une absorbance $\mathrm{A}_{660 \mathrm{~nm}}$ de 2,1 . Pour différentes concentrations de glutamate $(2,5,7 \mathrm{mM})$, le meilleur rendement de production $\mathrm{d}^{\prime} \mathrm{H}_{2}$ rapporté à la consommation de lactate est obtenu avec $5 \mathrm{mM}$ de glutamate dans le milieu nutritif. L'analyse du gaz produit, par spectrométrie de masse et chromatographie gazeuse, indique un taux d'hydrogène de lordre de $90 \%$, le reste étant du $\mathrm{CO}_{2}$ et de l'argon (gaz vecteur d'alimentation).

Ce résultat n'est pas en accord avec la réaction d'oxydation totale du lactate qui s'écrit :

$$
\mathrm{CH}_{3}-\mathrm{CHOH}-\mathrm{COOH}+3 \mathrm{H}_{2} \mathrm{O} \longrightarrow 3 \mathrm{CO}_{2}+6 \mathrm{H}_{2}
$$

et qui conduirait à un mélange contenant $33 \%$ de $\mathrm{CO}_{2}$ et $66 \%$ de $\mathrm{H}_{2}$. Or, les solubilités respectives de l'hydrogène et du dioxyde de carbone sont très différentes à $25^{\circ} \mathrm{C}$ :

[ $\mathrm{H}_{2}$ dissous] (en mole $\left.\cdot \mathrm{H}^{-1}\right)=7,810^{-4} \mathrm{P}_{\mathrm{H} 2}$

$\left[\mathrm{CO}_{2}\right.$ dissous] (en mole $\left.\cdot r^{-1}\right)=3,410^{-2} \mathrm{P}_{\mathrm{CO} 2}$

et à l'équilibre de dissolution s'ajoutent deux équilibres acide-base :

$\left[\mathrm{H}^{+}\right]\left[\mathrm{HCO}_{3}^{-}\right]=4,31^{-7} \mathrm{PCO}_{2}$

$\left.\left[\mathrm{H}^{+}\right]\left[\mathrm{CO}_{3}{ }^{2-}\right]=5,610^{-11}\left[\mathrm{HCO}_{3}{ }^{-}\right]=2,410^{-17} \mathrm{P}_{\mathrm{CO} 2} / \mathrm{H}^{+}\right]$

soit $\left[\mathrm{CO}_{2}\right.$ total $]=\mathrm{P}_{\mathrm{CO} 2}\left[3,410^{-2}+4,310^{-7} 1 \mathrm{OPH}^{\mathrm{pH}}+2,410^{-17} 10^{2 \mathrm{pH}}\right]$

donc $\mathrm{C}_{\mathrm{CO} 2}=\mathrm{P}_{\mathrm{CO} 2} \cdot \mathrm{F}(\mathrm{pH})$ 
Tableau 1 Variation de la production d'hydrogène, de la densité bactérienne, de la dégradation du lactate en fonction du taux de dilution. Le milieu entrant contient $25 \mathrm{mM}$ lactate, $7 \mathrm{mM}$ glutamate, $\mathrm{pH} 6,9$.

\begin{tabular}{|c|c|c|c|c|c|c|c|}
\hline $\begin{array}{c}D \\
\left(h^{-1}\right)\end{array}$ & $\begin{array}{c}\text { psb } \\
\left(q \cdot r^{1}\right)\end{array}$ & $\left(m \mid \cdot r^{-1} \cdot g^{-1} p s b\right)$ & $\begin{array}{c}\mathrm{H}_{2} \\
\left(\mathrm{mmol} \cdot \mathrm{h}^{-1} \cdot \mathrm{r}^{-1}\right)\end{array}$ & $\left(\left.\mathrm{mmol} \cdot\right|_{\mathrm{r}^{-1}} ^{-r_{\mathrm{s}}} \cdot \mathrm{r}^{-1}\right)$ & $\left(g \cdot n^{-1} \cdot r^{-1}\right)$ & $\begin{array}{c}Y_{x} \\
\left(0 \cdot Q_{-1}\right)\end{array}$ & $\begin{array}{c}Y_{\mathrm{H}_{2}} \\
\left(\mathrm{~mol}^{\prime} \cdot \mathrm{mot}^{-1}\right)\end{array}$ \\
\hline 0,026 & 1,12 & 34,5 & 1,74 & 0,75 & 0,029 & 0,43 & 2,32 \\
\hline 0,04 & 1,08 & 41 & 1,98 & 1,04 & 0,043 & 0,46 & 1,9 \\
\hline 0,05 & 1,02 & 38,2 & 1,74 & 1,25 & 0,051 & 0,45 & 1,39 \\
\hline 0,066 & 0,99 & 32 & 1,4 & 1,5 & 0,065 & 0,48 & 0,93 \\
\hline 0,096 & 0,94 & 15,3 & 0,65 & 1,8 & 0,09 & 0,55 & 0,36 \\
\hline 0,11 & 0,9 & 7,4 & 0,3 & 2,09 & 0,099 & 0,52 & 0,14 \\
\hline 0,14 & 0,81 & 0 & & & & & \\
\hline
\end{tabular}

\footnotetext{
$x$ : matière vivante ou poids sec bactérien (psb)

$\mathrm{H}_{2}$ : production d'hydrogène

$\mathrm{r}_{\mathfrak{c}}$ : vitesse de dégradation du lactate

$r_{x}$ : vitesse de formation de la matiere vivante

$Y_{x}$ : masse de matière vivante formée/masse de lactate dégradé

$Y_{H_{2}}$ : quantitié d'hydrogène formée/masse de lactate dégradé
} 


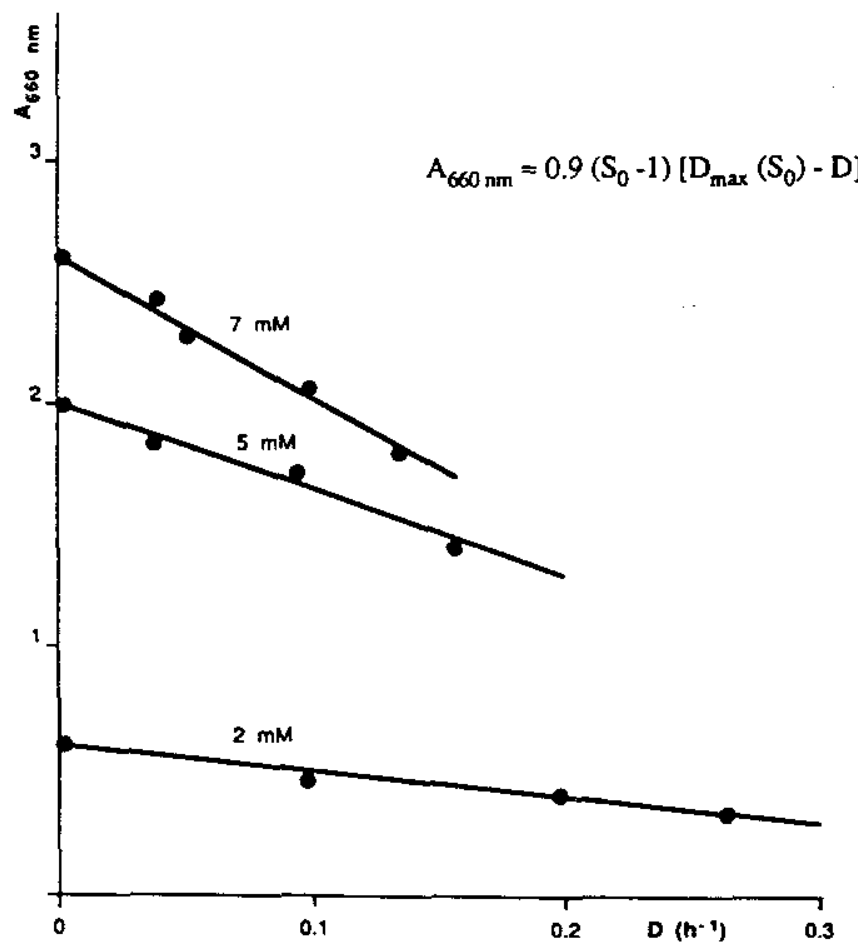

Flgure 5 Effet de la teneur initiale en glutamate $\left(S_{0}\right)$ sur la concentration bactérienne $\left(A_{660 \mathrm{~nm}}\right)$ en fonction du taux de dilution $D\left(\mathrm{~h}^{-1}\right)$. L'absorbance est reliée au psb par la relation psb $\left(\left.g \cdot\right|^{-1}\right)=0,45 \cdot A_{660 \mathrm{~nm}}$.

Ainsi, pour différentes valeurs du pH et pour $80 \mathrm{ml} \mathrm{de} \mathrm{H}_{2}$ formé par heure et par gramme de matière vivante, nous obtenons les résultats rassemblés dans le tableau 2 dans un bioréacteur contenant $0,6 \mathrm{~g}$ de biomasse par litre, alimenté à un taux de dilution de $\mathrm{D}=0,04 \mathrm{~h}^{-1}$ et où $\mathrm{C}_{\mathrm{CO} 2}=2,5 \cdot 10^{-2} \mathrm{~mole} \cdot \mathrm{F}^{-1}$. Par conséquent, pour un pH de plus en plus basique, la pression partielle de $\mathrm{CO}_{2}$ correspondant à la transformation totale de $25 \mathrm{mM}$ lactate, diminue. On estime alors que dans le mélange gazeux dégagé par les bactéries et pour un $\mathrm{pH}$ proche de la neutralité (nos conditions de culture), la pression partielle de $\mathrm{CO}_{2}$ gazeux ne peut dépasser $2 \%$.

Tableau 2 Pressions partielles de $\mathrm{CO}_{2}$ à l'équilibre pour des $\mathrm{pH}$ voisins de la neutralité.

\begin{tabular}{|lccc|}
\hline $\mathrm{PH}$ & 6,5 & 7 & 7,5 \\
$\mathrm{FpH})$ & 1,4 & 4,3 & 13,6 \\
$\mathrm{P}_{\mathrm{CO} 2}$ en $10^{-3} \mathrm{~atm}$ & 18,0 & 5,8 & 1,8 \\
\hline
\end{tabular}


La faible quantité de bicarbonate dissous dans la suspension, observée lors des bilans de carbone total et organique indique que le $\mathrm{CO}_{2}$ formé lors de la dégradation du lactate est réutilisé dans la synthèse de la biomasse (photoréduction du $\mathrm{CO}_{2}$ ) (voir DELACHAPELLE $\theta t$ al., 1990).

Nous avons vu qu'à des taux de dilution élevés $\left(>0,05 \mathrm{~h}^{-1} ;\right.$ tableau 1$)$ le rendement en hydrogène chute. En conséquence, une cellule d'ultrafiltration a été rajoutée au circuit pour recycler les bactéries dans la suspension circulante. Le recyclage permet d'augmenter la population bactérienne dans la suspension, de maintenir des temps de séjour importants et d'élever le débit d'alimentation en milieu nutritif. On retrouve alors les conditions idéales obtenues en culture continue (temps de séjour importants et dilution suffisante pour ne pas limiter la culture en énergie lumineuse) tout en augmentant le rendement du procédé.

\section{Fonctionnement en réacteur ouvert avec recyclage}

L'expérience consiste à recycler les bactéries dans le bioréacteur à travers une unité d'ultrafiltration. Le réacteur est alors alimenté en continu avec du milieu nutritif contenant $25 \mathrm{mM}$ de lactate et $2 \mathrm{mM}$ de glutamate. Lorsque l'état stationnaire est atteint, le système fonctionne en régime semi-ouvert (ultrafiltration) en alternant des cycles de recyclage $(100 \%)$ et de non recyclage. Deux taux de dilution sont choisis à $0,04 \mathrm{~h}^{-1}$ et $0,06 \mathrm{~h}^{-1}$, le $\mathrm{pH}$ est lui maintenu à 7,7 pendant toute la durée de l'expérience, grâce aux sondes de $\mathrm{pH}$ pressurisées et une régulation autonome parfaitement adaptée au système. La précision de la mesure est assurée avec un temps de réponse de $3,6 \mathrm{mn}$ correspondant à un débit de circulation du milieu dans le bioréacteur de $175 \mathrm{l} \cdot \mathrm{h}^{-1}$.

On a pu mettre en évidence un régime d'équilibre stable, correspondant à un fonctionnement du réacteur en continu, et un régime transitoire pendant les phases de recyclage et de retour de l'équilibre. Au cours du régime transitoire, la densité bactérienne augmente linéairement en fonction du temps, tant que la cellule d'ultrafiltration ne se colmate pas, puis décroît suivant une loi d'élution classique dans un réacteur ouvert parfaitement agité dès que le recyclage est arrêté. Le rapport du poids sec bactérien à l'absorbance mesurée à $660 \mathrm{~nm}$ est approximativement de 0,3 , puis s'élève à $0,4-0,5$ au cours du recyclage suite a la formation de matières de réserve et de polysaccharides qui tendent à accoler les bactéries les unes aux autres en chaîne. Ce phénoméne observé dans une culture à population bactérienne vieille et dense (temps de séjour important) est caractéristique d'une suspension recyclée.

La production d' $\mathrm{H}_{2}$ maximale $\left(70 \mathrm{ml} \cdot \mathrm{h}^{-1} \cdot \mathrm{g}^{-1} \mathrm{psb}\right)$ observée dans une culture peu dense (par exemple en système ouvert avec $2 \mathrm{mM}$ de glutamate) diminue, de même que l'activité de la nitrogénase, lors du fonctionnement en recyclage. Le recyclage s'accompagne d'une augmentation de la population bactérienne qui fait écran a la lumière, il y a donc moins bonne capture de l'énergie lumineuse par les bactéries. On observe alors un métabolisme de type fermentatif caractérisé par une plus faible dégradation du lactate avec accumulation d'acides organiques (acides acétique, propionique) dans la culture en phase de recyclage (voir DELACHAPELLE et al., 1990). De plus, 
après plusieurs recyclages successifs de la culture, l'activité nitrogénase diminue très lentement et la production d'hydrogène n'atteint plus la valeur maximale attendue $\left(80 \mathrm{ml} \cdot \mathrm{h}^{-1} \cdot \mathrm{g}^{-1} \mathrm{psb}\right)$.

\section{DISCUSSION}

Un réacteur pilote de 10 litres a été réalisé afin de permettre l'étude de la production $\mathrm{d}^{\prime} \mathrm{H}_{2}$ à partir d'un milieu synthétique contenant du lactate et du glutamate par la bactérie photosynthétique $R$. capsulatus. La réalisation a été définie à partir des besoins de croissance des bactéries favorisant une production d'hydrogène maximale. En particulier, les bactéries doivent être bien éclairées pour permettre une synthèse d'ATP à grande vitesse et l'oxygène doit être absent du milieu pour deux raisons : d'une part, parce que la nitrogénase responsable de la production d'hydrogène est inactive en présence de $\mathrm{O}_{2}$ et, d'autre part, parce que l'hydrogène produit par la nitrogénase est réoxydé en $\mathrm{H}_{2} \mathrm{O}$ par la chaîne respiratoire des bactéries (MEYER et al., 1978b ; PAUL ot al., 1979).

En système batch, sans limitation de substrats carbonés ou azotés, la culture peut être maintenue en croissance exponentielle et atteindre des valeurs élevées en biomasse. Nous avons ainsi obtenu des cultures contenant $5,4 \mathrm{~g}$ psb par litre $\left(A_{660 \mathrm{~nm}}=12\right)$. Mais au-delà d'une densité optique égale à 3 , la production d' $\mathrm{H}_{2}$ chute par diminution de l'activité de la nitrogénase liée à une limitation du transfert d'énergie lumineuse dans une culture trop dense et devenue opaque. Une augmentation de la production d'hydrogène allant de pair avec l'augmentation de l'intensité lumineuse a déjà été observée par JOUANNEAU et al. (1984) et STEVENS et al. (1984). C'est pourquoi, pour maintenir une bonne illumination des cellules, la croissance des bactéries a été limitée par limitation du substrat azoté.

La limitation en azote permet aussi une meilleure synthèse et une meilleure activité de la nitrogénase. Bien que l'ammoniaque, en concentrations millimolaires, réprime et inactive la nitrogénase, à faibles concentrations (de lordre du micromolaire) $\mathrm{NH}_{4}{ }^{+}$permet la production de $\mathrm{H}_{2}$ (JOUANNEAU et al., 1982 ; SEGERS et VERSTRAETE, 1985b). La croissance des Rhodospirillaceae sur $\mathrm{N}_{2}$ permet aussi une bonne induction de nitrogénase et par là une bonne conversion des acides organiques en $\mathrm{H}_{2}$ (SEGERS and VERSTRAETE, 1983). Néanmoins, la croissance est lente ot plus difficile à maîtriser dans un bioréacteur. C'est pourquoi le glutamate a été choisi comme source d'azote. On observe qu'une culture en continu a taux de dilution faible, $0,04 \mathrm{~h}^{-1}$. recevant un milieu nutritif contenant $5 \mathrm{mM}$ de glutamate, a une production maximale d'hydrogène de $85 \pm 10 \mathrm{ml} \cdot \mathrm{h}^{-1} \cdot \mathrm{F}^{-1}$, soit environ 2,1 litres par jour et par litre de culture, ce qui est supérieur aux productions maximales de $\mathrm{H}_{2}$, sur DL-lactacte, obtenues par STEVENS et al. (1983) dans leur photobioréacteur, avec les neuf souches de $R$. capsulatus que ces auteurs ont testé (qui ne comprenaient pas B10). 
Le bioréacteur pilote a permis de définir les conditions de culture permettant de maximiser la production d'hydrogène gráce a la régulation précise de paramètres clés ( $\mathrm{pH}$, température, taux de dilution). Le $\mathrm{pH}$ et le taux de dilution (en débits alimentation et soutirage) étaient régulés par l'intermédiaire de régulateurs analogiques autonomes PID. L'interdépendance de tous les paramètres variables rendant le système complexe, l'usage d'un système centralisé par ordinateur est à lheure actuelle inadapté et trop ambitieux pour une conduite entièrement automatisée de telles cultures bactériennes. En effet, ces micro-organismes sont capables de changer de métabolisme lorsque les conditions opératoires sont modifiées ; c'est ce que nous avons mis en évidence par l'utilisation d'une cellule d'ultrafiltration permettant d'augmenter la concentration bactérienne et le temps de séjour bactérien. Dans ces conditions, la production d'H $\mathrm{H}_{2}$ chute (fig. 4) en même temps qu'apparaissent des composés caractéristiques d'un processus de type fermentatif (propionate et acétate) (voir DELACHAPELLE et al., 1990).

\section{REMERCIEMENTS}

Ces recherches ont bénéficié de subventions de recherche du CNRS (PIRDES - URA 1130) et des Communautés Economiques Européennes (DG XII). Nous remercions vivement Mme BOYER pour sa contribution à la réalisation de cet article.

\section{RÉFÉRENCES BIBLIOGRAPHIQUES}

COLBEAU A., KELLEY B.C., VIGNAIS P. M., 1980. Hydrogeanse activity in Rhodopseudomonas capsulata: relationship with nitrogenase activity. J. Bacteriol., 144, 141148.

DELACHAPElle S., RENAUD $M$., VIGNAIS P.M., 1990. Etude de la production d'hydrogène par la bactérie photosynthétique Rhodobacter capsulatus. II. Transformation du lactate et bilans carbonés. Revue des Sciences de rEau (soumis).

JOUANNEAU Y., 1982. Etude de la fixation de razote et la photoproduction d'hydrogène chez la bactérie photosynthétique Rhodopseudomonas capsulata. Thèse de Doctorat d'Etat, Grenoble.
JOUANNEAU Y., WILLISON J.C., COLBEAU A., HALLENBECK P.C., RIOLACCI C., VIGNAIS P.M., 1982. Enhancement of the photoproduction of $\mathrm{H}_{2}$ by Rhodopseudomonas capsulata : optimization in contimoous culture, role of uptake hydrogenase, genetic characterization and economic evaluation. In : Photochemical, Photoelectrochemical and Photobiological Processes (D.O. Hall and W. Palz, ods), Solar Energy Research and Development in the European Community, Series D, vol. 1 , 174-179. Reidel, Dordrecht.

JOUANNEAU Y., LEBECQUE S., VIGNAIS P.M., 1984. Ammonia and light effect on nitrogenase activity in nitrogen-limited continuous cultures of Rhodopseudomo- 
nas capsulata. Role of glutamine synthetase. Arch. Microbiol., 139, 326-331.

JOUANNEAU Y., WONG B., VIGNAIS P.M., 1985. Stimulation by light of nitrogenase synthesis in cells of Rhodopseudomonas capsulata growing in $\mathrm{N}$-limited continurous cultures. Biochim. Biophys. Acta, 808, 149 155.

JOUANNEAU Y., THIERRY J.P., VIGNAIS P.M., RENAUD M., 1986. Epuration ot photoproduction d'hydrogene par des bactéries photosynthétiques. Entropie, 127. 18-19.

HALLENBECK P.C., 1983. Immobilized microorganisms for hydrogen and ammonia production. Enzyme Microb. Technol., 5, 171-180.

HILLMER P., GEST H., 1977. $\mathrm{H}_{2}$ metabolism in the photosynthetic bacterium Rhodopseudomonas capsulata: $\mathrm{H}_{2}$ production by growing cultures. J. Bacteriol. 129, 724731.

IMHOFF J.F., TRUPER H.G., PFENNIG N., 1984. Rearrangement of the species and genera of the phototrophic * purple non sulfur bacteria . J. Syst. Bacteriol. 34, 340-343.

LEVEAU J.Y., BOUIX M., 1982. Cinétiques microbiennes. In: Biotechnologie (R., Scriban, ed), 121-150, Lavoisier, Paris.

MEYER J., KELLEY B.C., VIGNAIS P.M., 1978a. Aerobic nitrogen tixation by $R$ hodopseudomonas capsulata. FEBS Lett., 85, 224-228.

MEYER J., KELLEY B.C., VIGNAIS P.M., $1978 \mathrm{~b}$. Nitrogen fixation and hydrogen metabolism in photosynthetic bacteria. Biochimie, 60, 245-260.

PAUL. F., COLBEAU A., VIGNAIS P.M., 1979. Phosphorylation coupled to $\mathrm{H}_{2}$ oxidation by chromatophores from Rhodopseudomonas capsulata. FEBS Lett. 106, 29-33.

SEGERS L., VERSTRAETE W., 1983. Conversion of organic acids to $\mathrm{H}_{2}$ by Rhodospirillaceae grown with ghutamate or dinitrogen as nitrogen source. Biotechnol. Bioeng., 25, 2843-2853.

SEGERS L., VERSTRAETE $W ., 1985 a$. Chemical control of eucaryotic and blue. green algae in anaerobic photoreactors culturing Rhodospirillaceae. Experientia 41, 99-101.

SEGERS L., VERSTRAETE $W ., 1985 \mathrm{~b}$. Ammonium as an alternative nitrogen source for hydrogen producing photobacteria. J. Appl. Bacteriol., 58, 7-11.

STEVENS P., VAN DER SYPT H., DE VOS P., DE LEY P., 1983. Comparative study on $\mathrm{H}_{2}$ evolution from DL lactate, acetate and butyrate by different strains of Rhodopseu. comonas capsulata in a new type of reactor. Biotechnol. Letters, 5, 369-374.

STEVENS P., VERTONGHEN C., DE VOS P., DE LEY J., 1984. The effect of temperature and light intensity on hydrogen gas production by different Rhodopseudomonas capsulata strains. Biotechnol. Letters, 6, 277-282.

VIGNAIS P.M., MEYER J., HALLENBECK P.C., 1981. Photoproduction of $\mathrm{H}_{2}$ by photosynthetic bacteria. HydrogenaseNitrogenase interrelationship. Postpy Biologii Komorki, 8, 141-161.

VIGNAIS P.M., WILLISON J.C., COLBEAU A., JOUANNEAU Y., MADERN D., HALLENBECK P.C., THIERRY J.P., RENAUD M., 1984. Photoproduction of $\mathrm{H}_{2}$ and $\mathrm{NH}_{3}$ by the photosynthetic bacterium Ahodopseudomonas capsulata. Energy-Commission of the European Communities, Report EUR 9440 EN, 1-24. EAEC Bruxelles, Luxembourg.

VIGNAIS P.M., COLBEAU A., WILLISON J.C., JOUANNEAU Y., 1985. Hydrogenase, nitrogenase and hydrogen metabolism in the photosynthetic bacteria. Adv. Microbiol. Physiol. 26, 155-234.

VINCENZINI M., BALLONI W., MANNELLI D., FLORENZANO G., 1981. A bioreactor for continuous treatment of waste waters with immobilized cells of photosynthetic bacteria. Experientia, 37, 710-711.

VINCENZINI M., MATERASSI R., TREDICI M.R., FLORENZANO G., 1982. Hydrogen production by immobilized cells - 1 - Light dependent dissimilation of organic substances by Rhodopseudomonas palustris. Int. J. Hyodrogen Energy. 7, 231-236.

WEAVER P.F., WALL J.D., GEST H., 1975. Characterization of Rhodopseudomonas capsulata. Archiv. Microbiol., 105, 207-216.

WEAVER P.F., LIEN S., SEIBERT M., 1980. Photobiological production of hydrogen. Solar Energy, 24, 3-45.

WILLISON J.C., JOUANNEAU Y., COLBEAU A., VIGNAIS P.M., 1983. $\mathrm{H}_{2}$ metabolism in photosynthetic bacteria and relationship to $\mathrm{N}_{2}$ fixation. Ann. Microbiol. (Inst. Pasteur) $143 B, 115-135$ 